\title{
Ethik und Patientenverfügung - zu viel des Guten?
}

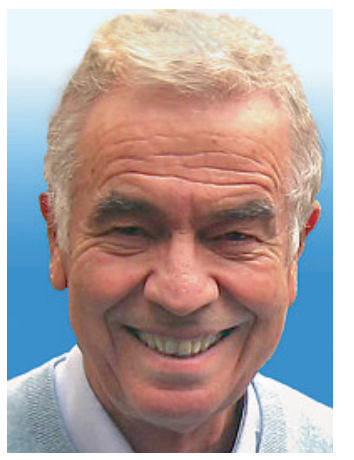

Hans Stalder
Ein merkwürdiger Unterschied: Der zurechnungsfähige Mensch kann immer auch anders, der unzurechnungsfähige nie!

Robert Musil

Die Nationale Ethikkommission (NEK) hat Stellung genommen zum neuen Schweizer Erwachsenenschutzrecht, das die Gültigkeit der Patientenverfügung (PV) bestärkt. Glücklicherweise gibt es eine Zusammenfassung in der Ausgabe 32/33 der Schweizerischen Ärztezeitung [1], denn die Lektüre des rund vierzig Seiten umfassenden Textes gestaltet sich eher mühsam. Die NEK begrüsst die juristische Neuerung, räumt jedoch auch einige Ausnahmen ein, darunter die Verweigerung der Schmerztherapie oder der Nahrungsmittelaufnahme. Dies erscheint eher willkürlich, denn warum sollte jemand nicht die Freiheit haben, Schmerzmittel oder Nahrung zu verweigern, selbst wenn dies gegen die Berufsethik der Pflegenden verstossen mag?

Es gibt jedoch Punkte, die mir problematischer erscheinen. Die NEK nennt drei Grundvoraussetzungen, damit eine Patientenverfügung wirklich verbindlich abgefasst ist. Eine davon ist die Urteilsfähigkeit - da wird jedermann zustimmen. Schwierigkeiten habe ich mit den anderen Voraussetzungen: der Antizipationsfähigkeit (im Rahmen der Urteilsfähigkeit) und der «Kontinuität der Person».

Wenn es schon schwierig ist, vorherzusagen, in welchem Zustand wir uns in drei Wochen befinden werden, so gestaltet sich dies noch viel heikler für den Fall einer ernsthaften Erkrankung - insbesondere einer fortgeschrittenen Demenz - die in Jahren eintreten kann (oder auch nicht) und möglicherweise von vielen anderen Umständen, wie dem Verlust einer nahestehenden Person oder altersbedingter Gebrechlichkeit, begleitet wird. Ein Verlust der Urteilsfähigkeit kann im Übrigen auch in vielen anderen Lebensumständen auftreten, zum Beispiel bei einer Hirnerschütterung oder einer Sepsis. Kann ich meinen Willen in Bezug auf die Versorgung für all diese Fälle antizipieren? Auch die Kommission gesteht zu, dass «immer eine Diskrepanz zwischen vorgestellter und gelebter Realität besteht und Entscheidungen nie in Kenntnis aller Informationen getroffen werden».

Noch problematischer wird es bei der dritten Grundvoraussetzung: «Die Kontinuität der Person ist eine normative Grundvoraussetzung für die Gültigkeit einer $P V »$. Dass jemand immer dieselbe Person bleibt, ist eine triviale Aussage. Die Kommission geht jedoch noch weiter. Sie geht davon aus, dass eine an Demenz erkrankte Person weiterhin als «derselbe biologische Organismus» fortbesteht. Das ist mehr als fragwürdig, denn alle Entwicklungen im Leben - Wachstum, Alterungsprozesse, Erkrankungen - gehen einher mit kon- tinuierlichen biologischen Veränderungen. Die Kommission postuliert weiter, dass «die Person weiterhin denselben Namen trägt» - trivial - und weiterhin «dieselben sozialen Rollen ausfüllt, beispielsweise Vater oder Schwester zu sein». Diese Aussage erstaunt: Sind es nicht gerade schwere Erkrankungen wie die Demenz, durch die sich die sozialen Rollen der Beteiligten tiefgreifend verändern, selbst wenn deren Position innerhalb der sozialen Struktur unverändert bleibt?

Robert Musil sagt in «Der Mann ohne Eigenschaften», dass das Privileg jener, die noch über ihre Urteilsfähigkeit verfügen, darin besteht, dass sie ihre Meinung ändern können - beispielsweise hinsichtlich einer neuen Situation und, warum nicht, nach einem Gespräch mit dem Arzt. Das ist ja die Problematik der PV im Fall eines Verlustes der Urteilsfähigkeit: Durch die Verpflichtung, den Inhalt der PV wortgetreu zu erfüllen, wie es die neue Gesetzgebung vorsieht, wird die Freiheit der Meinungsänderung jener Person, die ihre Urteilsfähigkeit verloren hat, eingeschränkt. Hier liegt auch das wirkliche ethische Problem. Indem wir uns auf eine Aussage vor der Krankheit fixieren, schliessen wir aus, dass sich der Patient an eine neue Situation, die seine Antizipationsfähigkeit nicht vorhergesehen hat, angepasst haben könnte. Unter dem Argument der Patientenautonomie, die vor dem Verlust der Urteilsfähigkeit vorliegt und danach nicht mehr dieselbe ist, laufen wir Gefahr, allfällige Willensänderungen der Betroffenen, die diese nicht mehr zum Ausdruck bringen können, zu vernachlässigen. Auf der einen Seite steht die PV - sie ist natürlich wichtig und muss berücksichtigt werden - auf der anderen Seite gilt es aber auch den akuten Gesundheitszustand, die veränderte soziale Rolle des Patienten zu berücksichtigen und weit schwieriger - die Möglichkeit zu erwägen, dass die betreffende Person ihre Meinung geändert haben könnte, wie ein freier Mensch, der über seine Urteilsfähigkeit verfügt. Für dieses Dilemma gibt es keine fertige Lösung, ganz sicher keine juristische. Da mag wohl nur echte Empathie der Ärzte und Pflegenden uns einer Lösung näherbringen.

Selbstverständlich müssen wir das Recht haben, unsere Wünsche zu äussern, was im Falle einer schweren Erkrankung mit uns geschehen soll, und solche Anweisungen auch umgesetzt zu sehen. PV sind ein wichtiges Argument in der medizinischen Entscheidungsfindung. Ich möchte aber auch das Recht behalten, meine Meinung zu ändern, selbst wenn ich sie nicht mehr ausdrücken kann!

War es wirklich notwendig, dass die Rechtsprechung sich einer so delikaten und intimen Thematik annimmt und die NEK dazu ihre umfangreiche Dialektik entwickelt?

Hans Stalder* 